\title{
Uso de Betabloqueador no Pós-Operatório de Cirurgia de Norwood Modificada por Sano com Cianose Persistente
}

\author{
Use of Beta-Blockers in the Post-Operative Period of Sano Modification of the Norwood Procedure with \\ Persistent Cyanosis
}

\author{
Eli Szwarc, Maria H. S. Rodrigues, Simone F. Pedra, Marcelo B. Jatene, Edson R. Romano, Carlos R. Ferreiro \\ Hospital do Coração - Associação do Sanatório Sírio - São Paulo, SP - Brasil
}

A cianose persistente constitui complicação freqüente no pós-operatório da cirurgia de Norwood modificada por Sano. Sua ocorrência pode ser explicada pela obstrução dinâmica da anastomose proximal do conduto sintético, que liga o ventrículo direito ao tronco pulmonar, evidenciada à ecocardiografia. A fim de melhorar a saturação arterial de oxigênio, foi recentemente descrita a utilização de betabloqueador, no período pós-operatório. Neste relato, descrevemos a utilização de propranolol em dois casos de cirurgia de Norwood modificada por Sano, demonstrando diminuição do gradiente no tubo sintético, aumento dos níveis de saturação arterial de oxigênio, diminuição da freqüência cardíaca e aumento da pressão arterial, traduzido em melhora clínica. Concluímos que a utilização de betabloqueador nesses casos mostrou-se benéfica.
Persistent cyanosis is a frequent complication in the post-operative period of Sano modification of the Norwood procedure. It may be explained by a dynamic proximal shunt stenosis of the synthetic conduit that links the right ventricle to the pulmonary artery, as detected by echocardiographic study. The use of beta-blockers in the post-operative period has been recently described in order to improve the arterial oxygen saturation. In this report, we describe the use of propranolol in two patients undergoing Sano modification of the Norwood procedure, in whom a gradient reduction in the synthetic conduit, increase in the levels of arterial oxygen saturation, decrease in heart rate, and increase in blood pressure were observed, thus resulting in clinical improvement. We conclude that the use of beta-blockers in these cases was beneficial.

\section{Introdução}

A síndrome de hipoplasia do ventrículo esquerdo (SHVE) é caracterizada por uma variedade de anormalidades cardíacas congênitas, que inclui hipodesenvolvimento acentuado do ventrículo esquerdo, hipoplasia ou atresia da valva aórtica e hipoplasia da aorta ascendente em graus variáveis, geralmente acompanhada por atresia de valva mitral e/ou coarctação de aorta. Sem intervenção cirúrgica a SHVE é fatal, com $25 \%$ de mortalidade na primeira semana de vida ${ }^{1}$. Historicamente, o tratamento da SHVE vem sendo conduzido de duas formas distintas: com cirurgias paliativas, que se baseiam na reconstrução da aorta ascendente (Norwood e variações), ou com transplante cardíaco.

Apesar de recentes avanços nas técnicas cirúrgicas para a reconstrução da aorta ascendente, os índices de mortalidade e de morbidade perioperatórias associadas com o primeiro estágio ainda permanecem muito elevados. Recentemente, Sano e cols. ${ }^{2}$ descreveram uma modificação na realização

\section{Palavras-chave}

Betabloqueadores, cirurgia de Norwood, cirurgia torácica, cianose, beta-antagonistas adrenérgicos, cardiopatias congênitas.

Correspondência: Eli Szwarc •

Rua Desembargador Eliseu Guilherme, 147 - Paraíso - 04004-030 - São Paulo, SP - Brasil

E-mail: eli.med@terra.com.br

Artigo recebido em 10/07/06; revisado recebido em 10/01/07; aceito em 26/02/07. do procedimento paliativo do primeiro estágio na SHVE, utilizando um conduto sintético de politetrafluoretileno (tubo VD-TP), que vai do ventrículo direito (VD) para o tronco pulmonar (TP), a fim de promover fluxo sangüíneo para os pulmões ao invés de realizar um shunt sistêmico-pulmonar tipo Blalock.

Os resultados preliminares dessa nova técnica mostraram-se favoráveis, mas a experiência ainda é limitada. Novas medidas terapêuticas baseadas em conhecimento tanto anatômico como fisiopatológico vêm sendo utilizadas, com o objetivo de melhorar a sobrevida de crianças submetidas ao primeiro estágio da cirurgia ${ }^{3}$. Uma intercorrência relativamente comum que temos presenciado nessas crianças é o surgimento de cianose acentuada no pós-operatório, com queda da saturação arterial de oxigênio para níveis abaixo de 70\%. Essa queda é atribuída à obstrução dinâmica que se apresenta em graus variáveis na anastomose proximal do tubo VD-TP, podendo ser observada à ecocardiografia.

No intuito de minimizar os efeitos dessa obstrução dinâmica, com diminuição do fluxo sangüíneo para a circulação pulmonar e conseqüente queda da saturação arterial de oxigênio, administramos betabloqueador (propranolol) a dois recém-nascidos, submetidos a cirurgia de Norwood modificada por Sano.

\section{Relato do Caso}

Dois pacientes recém-nascidos, portadores de SHVE, foram submetidos a cirurgia de Norwood modificada por Sano no terceiro e no décimo terceiro dias de vida, respectivamente. 
Ambos evoluíram com cianose persistente no período pósoperatório, mantendo saturação de oxigênio abaixo de $70 \%$. O grau de obstrução dinâmica na anastomose proximal do tubo VD-TP foi diagnosticado e estimado pela ecocardiografia bidimensional com Doppler à beira do leito. No sétimo e no nono dias de pós-operatório, respectivamente, os pacientes foram tratados com betabloqueador (propranolol) por via enteral, na dose de $1 \mathrm{mg} / \mathrm{kg} / \mathrm{dia}$, fracionado em duas vezes, e acompanhados clinicamente.

O paciente 1 , no início da terapia, recebia dobutamina $(10 \mu \mathrm{g} / \mathrm{kg} / \mathrm{min})$, milrinona $(0,45 \mu \mathrm{g} / \mathrm{kg} / \mathrm{min})$ e adrenalina $(0,03$ $\mu \mathrm{g} / \mathrm{kg} / \mathrm{min})$, permanecendo sedado e entubado, ventilando em modalidade pressão regulada com volume controlado ( $\mathrm{FR}=$

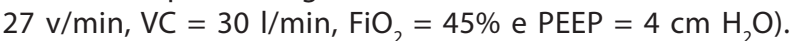
O paciente 2 , no início da terapia, recebia dobutamina $(9,5$ $\mu \mathrm{g} / \mathrm{kg} / \mathrm{min})$ e milrinona $(0,8 \mu \mathrm{g} / \mathrm{kg} / \mathrm{min})$, permanecendo em ventilação não-invasiva na modalidade CPAP nasal. Durante os três dias subseqüentes, não houve mudança significativa na prescrição de drogas vasoativas e nos parâmetros ventilatórios. Na tabela 1, estão descritas as medidas do gradiente do tubo VD-TP (antes e 72 horas após a terapia) e das médias das 72 horas anteriores à terapia e das subseqüentes das pressões arteriais sistólica, diastólica e média (aferidas a cada hora), além da saturação arterial de oxigênio (obtida da gasometria arterial colhida a cada seis horas), e o desvio padrão de todos os parâmetros.

\section{Resultados}

Os pacientes apresentaram gradiente de $42,8 \mathrm{mmHg}$ e $42 \mathrm{mmHg}$ (média de $42,4 \mathrm{mmHg}$ ) antes da terapia, e de 24 $\mathrm{mmHg}$ e $28 \mathrm{mmHg}$ (média de $26 \mathrm{mmHg}$ ) após 72 horas.

A saturação arterial de oxigênio antes da terapia foi de $63,9 \%$ e $66,1 \%$ (média de $65 \%$ ), e de $72,3 \%$ e $78,1 \%$ (média de $75,2 \%$ ) após a terapia. A freqüência cardíaca antes da terapia foi de 165 bpm e 153 bpm (média de 159 bpm), e de 142 bpm e 124,4 bpm (média de 133,2 bpm) após a terapia. A pressão arterial sistólica antes da terapia foi de $68,1 \mathrm{mmHg}$ e $82,5 \mathrm{mmHg}$ (média de 75,3 mmHg), e de $77,8 \mathrm{mmHg}$ e 90,3 $\mathrm{mmHg}$ (média de $84 \mathrm{mmHg}$ ) após a terapia. A pressão arterial diastólica antes da terapia foi de $48,9 \mathrm{mmHg}$ e $56,3 \mathrm{mmHg}$ (média de 52,6 mmHg), e de $50,7 \mathrm{mmHg}$ e $57,2 \mathrm{mmHg}$ (média de 53,9 mmHg) após a terapia. A pressão arterial média antes da terapia foi de $56,5 \mathrm{mmHg}$ e $65,7 \mathrm{mmHg}$ (média de $61,1 \mathrm{mmHg}$ ), e de $61 \mathrm{mmHg}$ e $68,9 \mathrm{mmHg}$ (média de 64,9 $\mathrm{mmHg}$ ) após a terapia.

\section{Discussão}

A SHVE é uma afecção que apresenta alta mortalidade, mesmo após tratamento cirúrgico. Sua abordagem pósoperatória deve ser individualizada para a técnica operatória utilizada, pois no procedimento clássico (cirurgia de Norwood) as circulações sistêmica e pulmonar estão em série, enquanto na técnica modificada por Sano encontram-se em paralelo. Além disso, na técnica modificada por Sano a utilização de tubo sintético na anastomose entre VD-TP pode apresentar obstrução dinâmica, levando a cianose persistente.

Este estudo demonstrou, em concordância com recente publicação de Simsic e cols. ${ }^{4}$, que o uso de betabloqueador no pós-operatório da cirurgia de Norwood modificada por Sano acarretou queda da freqüência cardíaca, aumento da saturação e diminuição do gradiente do tubo VD-TP. Embora este estudo não tenha sido desenhado para quantificar débito cardíaco e resistência vascular periférica, pode-se inferir clinicamente que houve aumento do débito cardíaco e diminuição da resistência vascular periférica. Com a melhora do desempenho cardíaco e a diminuição do grau de obstrução da anastomose proximal do tubo VD-TP, houve incremento da circulação pulmonar, com desvio da curva de dissociação da hemoglobina, proporcionando assim melhora significativa do quadro de cianose, com aumento da saturação arterial de oxigênio.

\section{Conclusão}

A estratégia terapêutica com o uso de propranolol mostrouse efetiva no tratamento da cianose grave causada pela obstrução dinâmica na anastomose proximal do tubo VD-TP após o procedimento de Norwood modificado por Sano.

Potencial Conflito de Interesses

Declaro não haver conflitos de interesses pertinentes.

Tabela 1 - Gradiente do tubo VD-TP e variáveis hemodinâmicas antes e 72 horas após a terapia com betabloqueador

\begin{tabular}{|c|c|c|c|c|c|c|c|c|}
\hline & \multicolumn{4}{|c|}{ Antes da terapia } & \multicolumn{4}{|c|}{ Após a terapia } \\
\hline & Caso 1 & Caso 2 & Média & $\mathrm{DP}$ & Caso 1 & Caso 2 & Média & $\mathrm{DP}$ \\
\hline Gradiente entre VD e TP (mmHg) & 42,8 & 42 & 42,4 & $\pm 0,5$ & 24 & 28 & 26 & $\pm 2,8$ \\
\hline Saturação arterial de oxigênio (\%) & 63,9 & 66,1 & 65 & $\pm 1,5$ & 72,3 & 78,1 & 75,2 & $\pm 4,1$ \\
\hline Freqüência cardíaca (bpm) & 165 & 153 & 159 & $\pm 8,4$ & 142 & 124,4 & 133,2 & $\pm 12,4$ \\
\hline Pressão arterial sistólica (mmHg) & 68,1 & 82,5 & 75,3 & $\pm 10,1$ & 77,8 & 90,3 & 84 & $\pm 8,8$ \\
\hline Pressão arterial diastólica $(\mathrm{mmHg})$ & 48,9 & 56,3 & 52,6 & $\pm 5,2$ & 50,7 & 57,2 & 53,9 & $\pm 4,5$ \\
\hline Pressão arterial média (mmHg) & 56,5 & 65,7 & 61,1 & $\pm 6,5$ & 61 & 68,9 & 64,9 & $\pm 5,5$ \\
\hline
\end{tabular}




\section{Relato de Caso}

\section{Referências}

1. Norwood WI, Kirklin JK, Sanders SP. Hypoplastic left heart syndrome: experience with palliative surgery. Am J Cardiol. 1980; 45: 87-91.

2. Sano S, Ishino K, Kawada M, Arai S, Kashara S, Asai T, et al. Right ventriclepulmonary shunt in first-stage palliation of hypoplastic left heart syndrome. JThorac Cardiovasc Surg. 2003; 126: 504-10.

3. De Oliveira NC, Ashburn DA, Khalid F, Burkhart HM, Adatia IT, Holtby HM, et al. Prevention of early sudden circulatory collapse after the Norwood operation. Circulation. 2004;110 (Suppl 1):II133-8.

4. Simsic JM, Cuadrado A, Kirshbom PM, Kanter KR, Ramaswamy D, Clabby $M$, et al. Novel management strategy for severe cyanosis after Sano modification of the Norwood procedure. J Thorac Cardiovasc Surg. 2005; 129 (6): 1450-1. 